\title{
The Effect of Periodontal Therapy on the Level of MMP-8 in Patients with Chronic Periodontitis
}

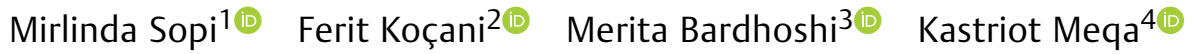 \\ ${ }^{1}$ Department of Periodontology and Oral Medicine, Dentistry School, \\ University of Pristina, Pristina, Kosovo \\ 2 Department of Dental Pathology and Endodontics, Dentistry School, \\ University of Pristina, Pristina, Kosovo \\ ${ }^{3}$ Department of Oro-Maxillofacial Surgery, University of Medicine, \\ Tirana, Tirana, Albania \\ ${ }^{4}$ Department of Periodontology and Oral Medicine, Dentistry School, \\ University of Pristina, Pristina, Kosovo \\ Eur J Dent 2023;17:70-75.

\begin{abstract}
Address for correspondence Kastriot Meqa, DDS, MSc, PhD, Department of Periodontology and Oral Medicine, Dentistry School, University of Pristina, Rrethi I Spitalit Street, NN, QKSUK, Pristina 10000, Kosovo (e-mail: kastriot.meqa@uni-pr.edu).
\end{abstract}

\author{
Abstract \\ Keywords \\ - chronic periodontitis \\ - diode laser \\ - periodontal flap \\ surgery \\ - matrix \\ metalloproteinase-8
}

Objective The complete removal of bacterial plaque and their endotoxins in deeper areas of periodontal pockets is often difficult to achieve with conventional methods such as periodontal pocket curettage. An alternative to these methods that recently gained popularity in periodontology is the diode laser, with its antibacterial effect, angiogenesis promotion as advantages.

Materials and Methods This study included 100 patients diagnosed with chronic periodontitis, with periodontal pockets up to $6 \mathrm{~mm}$, and who were divided into two groups: patients treated with basic therapy and diode laser application, and patients treated with basic therapy and with flap surgery. The clinical periodontal evaluation was done before the treatment and 6 months after the treatment. Evaluation of matrix metalloproteinase (MMP)-8 as an inflammatory indicator in gingival exudate was done with dipstick immunoassay test before the treatment and 6 months later.

Results This study showed a more pronounced improvement of periodontal clinical parameters, as well as a decrease in MMP- 8 values in gingival exudate in the lasertreated group compared with the surgically treated group.

Conclusion The results of this study are encouraging for the use of the diode laser as a noninvasive method in the treatment of periodontal pathologies.

\section{Introduction}

Periodontitis is a chronic inflammatory disease of bacterial etiology from dental plaque. ${ }^{1}$ Inflammation of the gingiva is a consequence of the initial lesions of the gingival epithelium caused by dental plaque microorganisms and triggering a range of reactions, such as cell infiltration, vascular changes, and changes in connective tissue elements and intercellular matter. ${ }^{1,2}$ It is important to detect initial signs of periodontitis for preventing the progression of this disease. ${ }^{3,4}$ Saliva is a reliable

article published online February 16, 2022
DOI https://doi.org/ $10.1055 / \mathrm{s}-0041-1742132$ ISSN 1305-7456. medium that reflects periodontal health and is easily accessible by identifying periodontal biomarkers. Thus, saliva is a very useful tool for monitoring not only oral but also systemic health. Periodontal tissue consists mainly of type I collagen. ${ }^{5-7}$ Periodontitis is characterized by loss of connective and bone tissues, which is mainly initiated by a group of enzymes called matrix metalloproteinases (MMPs) ${ }^{8,9}$ Therefore, laboratory detection of MMP-8 in gingival exudate enables differentiation of the inflammatory condition and degradation of periodontal tissues. ${ }^{10}$ The MMP family is divided into six groups of proteases:

\section{(c) 2022. The Author(s).}

This is an open access article published by Thieme under the terms of the Creative Commons Attribution License, permitting unrestricted use, distribution, and reproduction so long as the original work is properly cited. (https://creativecommons.org/licenses/by/4.0/)

Thieme Medical and Scientific Publishers Pvt. Ltd., A-12, 2nd Floor, Sector 2, Noida-201301 UP, India 
collagenases (MMP-1, MMP-8, and MMP-13), gelatinases (MMP-2 and MMP-9), stromelysins (MMP-3, MMP-10, and MMP- 11), matrixes (MMP-7 and MMP-26), MMPs of the type (MMP-14, MMP-15, MMP-16, MMP-17, and MMP-12), and other unclassified MMPs, given their auxiliary contrasts. ${ }^{11}$ MMP activities are inhibited and regulated by endogenous natural tissue inhibitors of matrix metalloproteinases (TIMPs) and $\alpha 2$-macroglobulin. Inequality between MMP and TIMP often results in devastating irreversible periodontal pathology. The proteolytic enzyme responsible for periodontal soft and hard tissues degeneration is the MMP-8, also known as collagenase- 2 or neutrophils. ${ }^{12-15}$ After the diagnosis, the treatment is planned, which includes all therapeutic procedures which are applied over a long period, and the success of the treatment is achieved by applying all the necessary actions. ${ }^{16,17}$ Traditional methods of treating periodontal injuries are mainly based on the removal of pathological contents from periodontal pockets mechanically through nonsurgical interventions-scaling and root planning. ${ }^{18-20}$ However, it has been argued that even after clinically successful healing, there is the possibility of reinfection by dental plaque biofilm residues. ${ }^{21-24}$ Therefore, in the last two decades, studies on semiconductor lasers or so-called diode lasers have been deepened to achieve a better therapeutic result. ${ }^{25-27}$ Clinical evidence to date points to the therapeutic advantages of using diode lasers as the first choice in the treatment of chronic periodontitis. ${ }^{28,29}$ The diode lasers have an advantage compared with the mechanical processing of periodontal tissue, as it removes all the altered epithelium then the biological stimulatory effect normalizes cell function, increasing microcirculation, stimulating fibroblasts, osteoblasts, odontoblasts, and increasing collagen formation. ${ }^{30}$ The laser also allows us better access to surfaces where it is anatomically difficult to reach with manual instruments. ${ }^{31}$ The study aimed to evaluate the level of MMP-8 in chronic periodontitis before and after treatment with two therapeutic methods: $980 \mathrm{~nm}$ diode laser and modified Widman flap (MWF).

\section{Materials and Methods}

This was a prospective randomized single-blinded clinical study. One hundred patients older than 18 years, with generalized (more than $30 \%$ of affected sites) stage III periodontitis (previously classified as generalized chronic periodontitis), with periodontal probing depth 4 to $6 \mathrm{~mm}$ deep, were included in this study. Excluded from the research were patients younger than 18 years, pregnant women, lactating women, patients currently treated with antibiotics for any other pathology, patients with addictions such as smoking, alcohol, and patients with parafunction. ${ }^{26}$ All patients were comprehensively informed about the content and purpose of the study before being part of the research. The study protocol was approved by the Ethics Committee of the Faculty of Medicine at the University of Prishtina under the Declaration of Helsinki.

\section{Evaluation of Periodontal Clinical Parameters}

Clinically, the condition of the periodontium was assessed using periodontal indices, such as pocket probing depth
(PPD), gingival index (GI), ${ }^{32}$ and clinical attachment level (CAL). ${ }^{33}$ These measurements were performed using a standardized periodontal probe on six surfaces of the examined dentition: mesiovestibular, vestibular, distovestibular, mezioral, oral, and distooral. To define the extent and severity of the periodontal disease, the updated classification of periodontal and peri-implant diseases and conditions was used. $^{34}$ Evaluation of clinical parameters was done before periodontal treatment, 3 and 6 months after.

\section{Matrix Metalloproteinase-8 Analysis}

The presence of bone destruction mediator MMP-8 was assessed by dipstick immunoassay test. The gingival exudate was taken from the periodontal pocket with the paper standing for 30 seconds, then placed in the buffer tube and the result was read after 5 minutes. Either positive or negative result was recorded. Evaluation of MMP-8 obtained from periodontal pocket exudate was done before treatment and 6 months after the treatment.

\section{Study Groups}

Patients were randomly divided into two groups:

Group I-Patients treated with basic therapy and diode laser application.

Group II-Patients treated with basic therapy and surgical procedure with flap.

The basic therapy for both groups consisted of debridement of supragingival hard and soft deposits, as well as scaling and root planning as a nonsurgical preparation for further interventions.

Laser therapy for patients of group I was performed using a diode laser (Laser HF, Hager-Werken, Germany) with the application of laser light inside the periodontal pocket of depth up to $6 \mathrm{~mm}$, and exposure of light of wavelength $980 \mathrm{~nm}$ with $10 \mathrm{~mW}$ power within 1 minute.

Standard surgical procedure using MWF for debridement of periodontal pockets was used for patients of group II. ${ }^{35}$ It was performed by a nonauthor of this article to exclude any bias.

\section{Statistical Analysis}

Data processing was done with the statistical package SPSS 22.0. The obtained data are presented in -Tables 1 to 8 .

\section{Results}

The research results show that both treatment methods have shown improvement compared with the baseline values before treatment. But the treatment with diode laser showed to be more effective in improvement of all clinical parameters, such as PPD, CAL, GI, and the level of MMP-8.

\section{Discussion}

The key to the successful treatment of periodontal disease is early diagnosis, which aids us to prevent disease progression, thus reducing the likelihood of periodontal tissues' loss. The 
72 Periodontal therapy and MMP-8 Sopi et al.

Table 1 CAL before the treatment in both groups of patients

\begin{tabular}{|l|l|l|}
\hline Baseline CAL & Laser treatment & Surgical treatment \\
\hline$N$ & 50 & 50 \\
\hline Average $(\mathrm{mm})$ & 4.42 & 4.38 \\
\hline SD & 1.03 & 0.99 \\
\hline SEM & 0.12 & 0.11 \\
\hline Minimum & 2.77 & 2.87 \\
\hline Maximum & 7.32 & 7.21 \\
\hline
\end{tabular}

Abbreviations: CAL, clinical attachment level; SD, standard deviation; SEM, standard error of the mean.

Note: Kruskal-Wallis' test, $p$-value $\mathrm{KW}=4.15, p=0.056$.

Table 2 CAL 6 months after the treatment in both groups of patients

\begin{tabular}{|l|l|l|}
\hline CAL after 6 mo & Laser treatment & Surgical treatment \\
\hline$N$ & 50 & 50 \\
\hline Average $(\mathrm{mm})$ & 3.18 & 3.29 \\
\hline SD & 0.89 & 0.83 \\
\hline SEM & 0.10 & 0.10 \\
\hline Minimum & 2.01 & 2.00 \\
\hline Maximum & 6.01 & 5.79 \\
\hline
\end{tabular}

Abbreviations: CAL, clinical attachment level; SD, standard deviation; SEM, standard error of the mean.

Note: Kruskal-Wallis' test, $p$-value $\mathrm{KW}=6.55, p=0.036$.

Table 3 PPD before the treatment in both groups of patients

\begin{tabular}{|l|l|l|}
\hline Baseline PPD & Laser treatment & Surgical treatment \\
\hline$N$ & 50 & 50 \\
\hline Average $(\mathrm{mm})$ & 4.18 & 4.14 \\
\hline SD & 0.66 & 0.58 \\
\hline SEM & 0.06 & 0.06 \\
\hline Minimum & 2.24 & 2.46 \\
\hline Maximum & 6.25 & 5.82 \\
\hline
\end{tabular}

Abbreviations: PPD, periodontal pocket depth; SD, standard deviation; SEM, standard error of the mean.

Note: Kruskal-Wallis' test, $p$-value $\mathrm{KW}=5.63, p=0.058$.

earlier diagnosis, the better prognosis of the disease. Following the evidence of scientific literature, worldwide experiences, and the need for less invasive treatment, this research evaluated the application of diode laser compared with the surgical method of treatment of periodontal disease and analyzed the level of MMP-8 in gingival exudate from the periodontal pocket.

In our surveyed patients before the treatment, around $80 \%$ of them appear to have the presence of MMP-8 in gingival exudate taken from the periodontal pocket, a strong correla-
Table 4 PPD 6 months after the treatment in both groups of patients

\begin{tabular}{|l|l|l|}
\hline PPD after 6 mo & Laser treatment & Surgical treatment \\
\hline$N$ & 50 & 50 \\
\hline Average $(\mathrm{mm})$ & 2.78 & 2.95 \\
\hline SD & 0.53 & 0.50 \\
\hline SEM & 0.06 & 0.06 \\
\hline Minimum & 2.00 & 2.00 \\
\hline Maximum & 5.73 & 5.64 \\
\hline
\end{tabular}

Abbreviations: PPD, periodontal pocket depth; SD, standard deviation; SEM, standard error of the mean.

Note: Kruskal-Wallis' test, $p$-value $\mathrm{KW}=26.8, p<0.0001$.

Table $5 \mathrm{Gl}$ before the treatment in both groups of patients

\begin{tabular}{|l|l|l|}
\hline Baseline Gl & Laser treatment & Surgical treatment \\
\hline$N$ & 50 & 50 \\
\hline Average (0-3) & 2.07 & 2.10 \\
\hline SD & 0.26 & 0.33 \\
\hline SEM & 0.03 & 0.04 \\
\hline Minimum & 1.24 & 1.02 \\
\hline Maximum & 3.00 & 3.00 \\
\hline
\end{tabular}

Abbreviations: GI, gingival index; SD, standard deviation; SEM, standard error of the mean.

Note: Kruskal-Wallis' test, $p$-value $\mathrm{KW}=2.55, p=0.235$.

Table 6 Gl 6 months after the treatment in both groups of patients

\begin{tabular}{|l|l|l|}
\hline Gl after $\mathbf{6}$ mo & Laser treatment & Surgical treatment \\
\hline$N$ & 50 & 50 \\
\hline Average $(0-3)$ & 0.16 & 0.28 \\
\hline SD & 0.40 & 0.45 \\
\hline SEM & 0.04 & 0.05 \\
\hline Min & 0.00 & 0.00 \\
\hline Max & 2.33 & 2.20 \\
\hline
\end{tabular}

Abbreviations: $\mathrm{Gl}$, gingival index; SD, standard deviation; SEM, standard error of the mean.

Note: Kruskal-Wallis' test, $p$-value $\mathrm{KW}=11.0, p=0.004$.

tion between clinical periodontal parameters and the presence of MMP-8 in gingival exudate. Research by Räisänen et al (2021) demonstrated the association between periodontal diagnostic clinical parameters (PPD) and the presence of MMP-8 in gingival exudate in patients with chronic periodontitis. $^{31}$

In our research, all analyzed periodontal clinical parameters improved in 6 months after laser treatment compared with patients who were treated with periodontal surgery, but with statistically important significance were PPD and CAL. In the MMP-8 assessment at 6 months, 96\% of laser 
Table 7 MMP- 8 before the treatment in both groups of patients

\begin{tabular}{|l|l|l|}
\hline Baseline MMP-8 & Laser treatment & Surgical treatment \\
\hline$N$ & $50(\%)$ & $50(\%)$ \\
\hline Negative & $10(20)$ & $9(18)$ \\
\hline Positive & $40(80)$ & $41(82)$ \\
\hline Total & $50(100)$ & $50(100)$ \\
\hline
\end{tabular}

Abbreviations: MMP-8, matrix metalloproteinase-8; SD, standard deviation; SEM, standard error of the mean.

Note: Chi-square test, $p$-value chi-square $=0.07, p=0.7988$.

Table 8 MMP-8 6 months after the treatment in groups of patients

\begin{tabular}{|l|l|l|}
\hline $\begin{array}{l}\text { MMP-8 after } \\
6 \text { mo }\end{array}$ & Laser treatment & Surgical treatment \\
\hline$N$ & $50(\%)$ & $50(\%)$ \\
\hline Negative & $48(96)$ & $40(80)$ \\
\hline Positive & $2(4)$ & $10(20)$ \\
\hline Total & $50(100)$ & $50(100)$ \\
\hline
\end{tabular}

Abbreviations: MMP-8, matrix metalloproteinase-8; SD, standard deviation; SEM, standard error of the mean.

Note: Chi-square test, $p$-value chi-square $=6.06, p=0.0138$.

treatment cases were negative compared with cases treated with surgery (80\%).

Khan et al (2021) evaluated the adjunct effect of $980 \mathrm{~nm}$ diode laser treatment of periodontal surgical therapy (MWF) for the treatment of chronic periodontitis. Periodontal surgical therapy together with laser therapy as an adjunct method led to a significant improvement of clinical parameters such as periodontal pocket depth, CAL, and better bactericidal effect 3 months after the treatment. ${ }^{36}$

Sezen et al (2020) evaluated the clinical and biochemical efficacy of laser treatment (Er, Cr: YSGG) compared with nonsurgical periodontal treatment, in patients with periodontitis. Both treatment modalities resulted in significant improvements in clinical parameters, but laser treatment was shown to be more effective in reducing periodontal inflammation. There were no statistically significant differences in interleukin (IL)-1 $\beta$ and MMP- 8 levels between the groups $(p<0.05){ }^{37}$

Deshmukh et al (2018) in their study evaluated the clinical and microbiological parameters, comparing the efficiency of periodontal treatment with diode laser and periodontal flap surgery treatment. The laser-treated group was found to have a higher decrease in the depth of periodontal pockets compared with the surgically treated group. The bactericidal effect of the laser was also significantly clearer in the reduction of periopathogens compared with the group treated with flap surgery. ${ }^{38}$

Karthikeyan et al (2019) in their research analyzed the effect of diode laser treatment as adjunctive therapy with Kirkland flap surgery. Diode laser treatment as adjunctive therapy in Kirkland surgery has resulted in a greater reduction of clinical and microbiological parameters compared with Kirkland surgery alone, thus offering additional benefits in treating patients with chronic periodontitis. $^{39}$

Saglam et al (2014) in their research on the clinical and biochemical effects of diode laser in the treatment of periodontitis showed significant improvements in clinical parameters and reduction of the level of MMP-8 in lasertreated patients compared with the group who were treated with scaling and root planning (SRP) only. ${ }^{40}$

Lobo and Pol (2015) investigated the adjunct effect of diode laser compared with periodontal surgical treatment, based on clinical parameters (periodontal pocket depth, $\mathrm{CAL}$, gingival atrophy, plaque index, GI, and tooth mobility) in early, 3, and 6 months after treatment. Their results showed a significant improvement of all periodontal clinical parameters after the therapy, but no statistically significant difference was encountered between the two treatment groups, except for a more noticeable reduction in gingival inflammation in the laser treatment group. Generally, patients regarded the laser treatment as more acceptable. $^{41}$

\section{Conclusion}

Concerning the periodontal clinical parameters of this research, it can be concluded that in patients treated with diode laser, there was an increase in CAL 3 and 6 months after treatment, a decrease in the depth of the periodontal pocket after 6 months, and a decrease of the GI, compared with the group treated with periodontal surgery. About the biological mediator of bone destruction, we have observed a decrease in the level of MMP-8 with a statistically significant difference in the cases treated with laser compared with the group treated with surgery. Based on these results, it would be interesting to extend the application of laser in the treatment of chronic periodontitis and apply clinical methods and protocols of this advanced technology.

\section{Authors' Contributions}

M.S., F.K., and K.M. conceptualized the study; methodology, formal analysis, investigation, resources, data curation, writing-original draft preparation, and funding acquisition were done by M.S.; software operated by K.M.; validation was done by M.S., F.K., M.B., and K.M.; writing-review and editing was done by F.K. and K.M.; visualization by K.M.; supervision by F.K. All authors have read and agreed to the published version of the manuscript.

\section{Ethical Approval}

This study was conducted according to the guidelines of the Declaration of Helsinki and approved by the Institutional Ethics Committee of the Faculty of Medicine of the University of Prishtina (protocol code 3833, May 12, 2017). 
Informed Consent

Informed consent was obtained from all subjects involved in the study.

\section{Funding}

None.

\section{Conflict of Interest}

None declared.

\section{References}

1 Baehni P, Tonetti MSGroup 1 of the European Workshop on Periodontology. Conclusions and consensus statements on periodontal health, policy and education in Europe: a call for actionconsensus view 1. Consensus report of the 1st European Workshop on Periodontal Education. Eur J Dent Educ 2010;14 (Suppl 1):2-3

2 Buduneli N, Kinane DF. Host-derived diagnostic markers related to soft tissue destruction and bone degradation in periodontitis. J Clin Periodontol 2011;38(Suppl 11):85-105

3 Franco C, Patricia HR, Timo S, Claudia B, Marcela H. Matrix metalloproteinases as regulators of periodontal inflammation. Int J Mol Sci 2017;18(02):440

4 Baeza M, Garrido M, Hernández-Ríos P, et al. Diagnostic accuracy for apical and chronic periodontitis biomarkers in gingival crevicular fluid: an exploratory study. J Clin Periodontol 2016;43(01): 34-45

5 Chapple ILC, Mealey BL, Van Dyke TE, et al. Periodontal health and gingival diseases and conditions on an intact and a reduced periodontium: consensus report of workgroup 1 of the 2017 World Workshop on the Classification of Periodontal and PeriImplant Diseases and Conditions. J Periodontol 2018;89(Suppl 1): S74-S84

6 Ramenzoni LL, Lehner MP, Kaufmann ME, Wiedemeier D, Attin T, Schmidlin PR. Oral diagnostic methods for the detection of periodontal disease. Diagnostics (Basel) 2021;11(03):571

7 Al-Majid A, Alassiri S, Rathnayake N, Tervahartiala T, Gieselmann D-R, Sorsa T. Matrix metalloproteinase- 8 as an inflammatory and prevention biomarker in periodontal and peri-implant diseases. Int J Dent 2018;2018:7891323

8 Sorsa T, Alassiri S, Grigoriadis A, et al. Active MMP-8 (aMMP-8) as a grading and staging biomarker in the periodontitis classification. Diagnostics (Basel) 2020;10(02):61

9 Sereti M, Roy M, Zekeridou A, Gastaldi G, Giannopoulou C. Gingival crevicular fluid biomarkers in type 1 diabetes mellitus: a case-control study. Clin Exp Dent Res 2021;7(02):170-178

10 Zhang F, Liu E, Radaic A, et al. Diagnostic potential and future directions of matrix metalloproteinases as biomarkers in gingival crevicular fluid of oral and systemic diseases. Int J Biol Macromol 2021;188:180-196

11 Öztürk VÖ, Emingil G, Umeizudike K, et al. Evaluation of active matrix metalloproteinase-8 (aMMP-8) chair-side test as a diagnostic biomarker in the staging of periodontal diseases. Arch Oral Biol 2021;124:104955

12 Teles R, Sakellari D, Teles F, et al. Relationships among gingival crevicular fluid biomarkers, clinical parameters of periodontal disease, and the subgingival microbiota. J Periodontol 2010;81 (01):89-98

13 Lorenz K, Keller T, Noack B, Freitag A, Netuschil L, Hoffmann T. Evaluation of a novel point-of-care test for active matrix metalloproteinase-8: agreement between qualitative and quantitative measurements and relation to periodontal inflammation. J Periodontal Res 2017;52(02):277-284

14 Izadi Borujeni S, Mayer M, Eickholz P. Activated matrix metalloproteinase-8 in saliva as diagnostic test for periodontal disease?
A case-control study. Med Microbiol Immunol (Berl) 2015;204 (06):665-672

15 Alshareef A, Attia A, Almalki M, et al. Effectiveness of probiotic lozenges in periodontal management of chronic periodontitis patients: clinical and immunological study. Eur J Dent 2020;14 (02):281-287

16 Hatipoğlu M, Barutcigil Ç, Harorlı OT, Ulug B. Effect of the lasers used in periodontal therapy on the surfaces of restorative materials. Scanning 2016;38(03):227-233

17 Mills MP, Rosen PS, Chambrone L, et al. American Academy of Periodontology best evidence consensus statement on the efficacy of laser therapy used alone or as an adjunct to non-surgical and surgical treatment of periodontitis and peri-implant diseases. J Periodontol 2018;89(07):737-742

18 Mizutani K, Aoki A, Coluzzi D, et al. Lasers in minimally invasive periodontal and peri-implant therapy. Periodontol 2000 2016;71 (01):185-212

19 Behdin S, Monje A, Lin GH, Edwards B, Othman A, Wang HL. Effectiveness of laser application for periodontal surgical therapy: systematic review and meta-analysis. J Periodontol 2015;86 (12):1352-1363

20 Kim TS, Schenk A, Lungeanu D, Reitmeir P, Eickholz P. Nonsurgical and surgical periodontal therapy in single-rooted teeth. Clin Oral Investig 2007;11(04):391-399

21 Gokhale SR, Padhye AM, Byakod G, Jain SA, Padbidri V, Shivaswamy $S$. A comparative evaluation of the efficacy of diode laser as an adjunct to mechanical debridement versus conventional mechanical debridement in periodontal flap surgery: a clinical and microbiological study. Photomed Laser Surg 2012;30(10): 598-603

22 Agarwal A, Saxena A, Gummaluri SS, Chaudhary B, Subramanyam S Sai K, Kumar G. Clinical and microbiological evaluation of 940$\mathrm{nm}$ diode laser as an adjunct to modified Widman flap for the management of chronic periodontitis: a 6-month randomized split-mouth clinical trial. J Dent Res Dent Clin Dent Prospect 2021; 15(02):133-139

23 Crespi R, Capparè P, Gherlone E, Romanos GE. Comparison of modified Widman and coronally advanced flap surgery combined with Co2 laser root irradiation in periodontal therapy: a 15-year follow-up. Int J Periodontics Restorative Dent 2011;31(06): 641-651

24 Sanz-Martín I, Cha JK, Yoon SW, Sanz-Sánchez I, Jung UW. Longterm assessment of periodontal disease progression after surgical or non-surgical treatment: a systematic review. J Periodontal Implant Sci 2019;49(02):60-75

25 Sobouti F, Khatami M, Heydari M, Barati M. The role of low-level laser in periodontal surgeries. J Lasers Med Sci 2015;6(02):45-50

26 Nevins M, Kim S-W, Camelo M, Martin IS, Kim D, Nevins M. A prospective 9-month human clinical evaluation of Laser-Assisted New Attachment Procedure (LANAP) therapy. Int J Periodontics Restorative Dent 2014;34(01):21-27

27 Akbulut N, Kursun ES, Tumer MK, Kamburoglu K, Gulsen U. Is the 810-nm diode laser the best choice in oral soft tissue therapy? Eur J Dent 2013;7(02):207-211

28 Rams TE, Loesche WJ. Relationship between periodontal screening and recording index scores and need for periodontal access surgery. J Periodontol 2017;88(10):1042-1050

29 Ali NM, Gujjari SK, Sankar AR. Open flap debridement using $810 \mathrm{~nm}$ diode laser and conventional surgery in patients on low dose aspirin- a comparative study. IOSR J Dent Med Sci 2019; 18:69-83

30 Harris DM, Nicholson DM, McCarthy D, et al. Change in clinical indices following laser or scalpel treatment for periodontitis: a split-mouth, randomized, multi-center trial. Proc SPIE Int Soc Opt Eng 2014;8929:89290G-1

31 Räisänen IT, Lähteenmäki H, Gupta S, et al. An aMMP-8 point-ofcare and questionnaire based real-time diagnostic toolkit for medical practitioners. Diagnostics (Basel) 2021;11(04):711 
32 Loe H, Silness J. Periodontal disease in pregnancy. I. Prevalence and severity. Acta Odontol Scand 1963;21:533-551

33 Ramfjord SP, Nissle RR, Shick RA, Cooper H Jr. Subgingival curettage versus surgical elimination of periodontal pockets. J Periodontol 1968;39(03):167-175

34 Tonetti MS, Greenwell H, Kornman KS. Staging and grading of periodontitis: framework and proposal of a new classification and case definition. J Periodontol 2018;89(Suppl 1):S159-S172

35 Ramfjord SP, Nissle RR. The modified Widman flap. J Periodontol 1974;45(08):601-607

36 Khan F, Chopra R, Sharma N, Agrawal E, Achom M, Sharma P. Comparative evaluation of the efficacy of diode laser as an adjunct to modified Widman flap surgery for the treatment of chronic periodontitis: a randomized split-mouth clinical trial. J Indian Soc Periodontol 2021;25(03):213-219

37 Sezen D, Hatipoğlu M, Üstün K. Evaluation of the clinical and biochemical efficacy of erbium, chromium:ytrium-scandium- gallium-garnet (ER,CR:YSGG) laser treatment in periodontitis. Lasers Med Sci 2020;35(07):1567-1575

38 Deshmukh K, Shetty D, Shetty A, Bodduru R, Nayyar AS. Comparative evaluation of the efficacy of closed pocket debridement with diode laser and periodontal open flap debridement: a clinical and microbiologic study. J Clin Sci 2018;15:113-122

39 Karthikeyan J, Vijayalakshmi R, Mahendra J, et al. Diode laser as an adjunct to Kirkland flap surgery-a randomized split-mouth clinical and microbiological study. Photobiomodul Photomed Laser Surg 2019;37(02):99-109

40 Saglam M, Kantarci A, Dundar N, Hakki SS. Clinical and biochemical effects of diode laser as an adjunct to nonsurgical treatment of chronic periodontitis: a randomized, controlled clinical trial. Lasers Med Sci 2014;29(01):37-46

41 Lobo TM, Pol DG. Evaluation of the use of a $940 \mathrm{~nm}$ diode laser as an adjunct in flap surgery for treatment of chronic periodontitis. J Indian Soc Periodontol 2015;19(01):43-48 